Recebido em 11/2019. Aceito para publicação em 12/2019.

\title{
ASPECTOS DA COMPOSIÇÃO DO BIODIESEL E SUA RELAÇÃO COM A RESISTÊNCIA A CORROSÃO DE LIGAS DE ALUMÍNIO
}

\author{
BIODIESEL COMPOSITION ASPECTS AND THEIR RELATION TO ALUMINUM \\ ALLOY CORROSION
}

\author{
Susan Melo da Rocha ${ }^{1}$ \\ Elisa Pereira de Carvalho ${ }^{2}$ \\ Nathalia Cerqueira da Silva ${ }^{3}$ \\ Rodrigo França Pacheco ${ }^{4}$ \\ Laerte Soares Filho ${ }^{5}$ \\ Roberta Gaidzinski ${ }^{6}$ \\ Neyda de la Caridad Om Tapanes ${ }^{7}$ \\ Ana Isabel de Carvalho Santana ${ }^{8}$
}

Resumo: As ligas de alumínio vêm sendo aplicadas na indústria de transporte e automobilística em virtude de propriedades tais como leveza e resistência à corrosão. Os biocombustíveis estão entre as alternativas avaliadas para substituir os combustiveis fósseis, pois apresentam como vantagem a biodegradabilidade e são provenientes de fontes renováveis. Esse trabalho realizou uma avaliação da resistência a corrosão de ligas de alumínio em biodiesel de soja, com diferentes concentrações de ácido oleico através de ensaios gravimétricos. Observou-se que a taxa de corrosão das ligas em biodiesel de soja puro é mais significativa nas primeiras horas de imersão, e apresenta uma significativa diminuição em tempos mais longos. Amostras de biodiesel de soja com teor de $20 \%$ de ácido oleico aumentaram a corrosão da liga de alumínio 6061 em comparação com amostras imersas em biodiesel puro.

Palavras-chave: Ligas de alumínio; biodiesel; corrosão; ácido oleico; ensaios gravimétricos.

Abstract: Aluminum alloys have been applied in the transportation and automotive industry due to properties such as lightness and corrosion resistance. Biofuels are a potential alternative to replace fossil fuels, because they are biodegradable and come from renewable sources. This work evaluated the corrosion resistance of aluminum alloys in soybean biodiesel, with different oleic acid concentrations. Aluminum corrosion behavior was obtained by gravimetric tests. It was observed that the corrosion rate of the alloys in pure soybean biodiesel is more significant in the first hours of immersion and presents a significant decrease in longer periods. Samples of soybean biodiesel with $20 \%$ oleic acid increased the corrosion of aluminum alloy 6061 compared to samples immersed in pure biodiesel.

Keywords: Aluminum alloys; biodiessel; corrosion; oleic acid; gravimetric tests.

\footnotetext{
1 Graduanda em Engenharia Metalúrgica, Escola de Engenharia, UEZO, Brasil. E-mail: susanmelodarocha@gmail.com.

2 Engenheira de Produção, Pós-graduanda PPGCTM, UEZO, Brasil. E-mail: elisa.carvalho@sh.com.br.

3 Tecnóloga em Construção Naval, Pós-graduanda PPGCTM, UEZO, Brasil. E-mail: nathaliacerqueiradasilva@hotmail.com.

4 Graduando em Engenharia Metalúrgica, Escola de Engenharia, UEZO, Brasil. E-mail: rfpachecho2010@hotmail.com.

5 Graduando em Engenharia Metalúrgica, Escola de Engenharia, UEZO, Brasil. E-mail: laertesoaresfilho@gmai.com.

6 Doutora em Engenheira Metalúrgica e de Materiais, Professora Adjunto, UEZO, Brasil. E-mail: rgaidzinski@ig.com.br.

7 Doutora em Engenharia Química, Professora Adjunto, UEZO, Brasil. E-mail: neydaom@yahoo.com.

8 Doutora em Engenharia Metalúrgica e de Materiais, Professora Adjunto, UEZO, Brasil: E-mail: isabelcarvalho.uezo@gmail.com.
} 


\section{INTRODUÇÃO}

As ligas de alumínio estão inseridas no grupo de materiais metálicos não-ferrosos e vêm sendo amplamente utilizadas em virtude da sua leveza, boa condutibilidade elétrica e térmica e reciclabilidade. As ligas de alumínio são geralmente utilizadas na indústria automobilística, naval, na construção civil, na indústria têxtil e também na indústria alimentícia. De uma forma geral, as ligas de alumínio possuem como elementos de liga: magnésio, manganês, cromo, ferro, silício e em alguns casos titânio (ALUMICUPPER, 2019).

De acordo com a Associação Brasileira de Alumínio - ABAL, atualmente se observa um crescimento na demanda pelas ligas de alumínio sobretudo no mercado de transporte, energia e construção civil. O aumento na indústria de transportes (veículos de passeio, caminhões, ônibus etc.) se deve principalmente à leveza do alumínio que promove um aumento da segurança, melhoria no desempenho e no consumo de combustíveis, além de promover uma redução da emissão dos gases de efeito estufa. Um exemplo da aplicação na indústria automotiva é na produção de peças tais como motores, câmbio e eixos em virtude da elevada condutividade térmica e da boa resistência à corrosão (ASSOCIAÇÃO BRASILEIRA DO ALUMíNIO, 2017). Outro exemplo é a aplicação da liga 5052 na produção de tanques de combustível (DEYAB, et al. 2019; OZTURK; TOROS; KILIC, 2008). Projeções recentes para o setor automotivo indicam um aumento de $30 \%$ do consumo de alumínio, sobretudo para países que já possuem regulamentações para limites de emissões e programas de mobilidade sustentável (ASSOCIAÇÃO BRASILEIRA DO ALUMÍNIO, 2017). Tendo em vista a previsão do aumento da demanda no consumo de alumínio e a crescente aplicação das ligas de alumínio na indústria automobilística, é importante conhecer as propriedades desses materiais tais como resistência mecânica e susceptibilidade à corrosão em situações que simulem diferentes condições ambientais.

A corrosão corresponde ao um processo de degradação dos materiais metálicos, que pode ocorrer por via química ou eletroquímica. A deterioração dos metais em virtude dos processos corrosivos é provocada através da interação entre o meio ambiente (oxidante) e o material. Os fenômenos corrosivos afetam diretamente o desempenho e a durabilidade dos materiais e em consequência disto provocam prejuízos de ordem econômica e ambiental. Estima-se que nos Estados Unidos o custo direto da corrosão em meados de 2002 era da ordem de 3,1\% do PIB (aproximadamente 276 bilhões de dólares) além de 552 bilhões em custos indiretos (KOCH et al., 2006; GENTIL, 2007; NUNES, 2007), no Brasil estimou-se em 2015 que os custos gerados pela corrosão atingiram em torno de 236 bilhões (aproximadamente 4\% do PIB). Dados mais recentes, avaliam que o impacto econômico global da corrosão se aproxima da ordem de 2,5 trilhões de dólares, um custo equivalente a aproximadamente $3,4 \%$ do PIB mundial (KOCH et al., 2016).

Em virtude do crescimento da aplicação das ligas de alumínio na indústria de 
transporte e automobilística é importante conhecer o comportamento destes materiais em contato com os diferentes tipos de combustíveis utilizados. Atualmente, considerando os conceitos de sustentabilidade e a preocupação com o meio ambiente, os biocombustíveis vêm sendo avaliados como possibilidade de substituição dos combustíveis fósseis.

O biodiesel é um dos potenciais candidatos como fonte de energia alternativa a matriz energética dos combustíveis fósseis pois possui algumas vantagens tais como a baixa toxicidade, é renovável e biodegradável além de apresentar um baixo teor de enxofre (FAZAL; HASEEB; MASJUKI, 2011; JIN et al., 2015; ALVES; DUTRA-PEREIRA; BICUDO, 2019; DEYAB et al., 2019). Entretanto, em comparação com os combustíveis fósseis, o biodiesel apresenta uma tendência em absorver água da atmosfera, possui uma menor estabilidade em altas temperaturas e apresenta processos de auto-oxidação que podem torná-lo mais corrosivo em comparação, por exemplo, com o diesel e outros combustíveis de origem fóssil (JIN et al., 2015, FERNANDES et al., 2013; CURSARU et al., 2014). Alguns trabalhos apontam que além das características higroscópicas do biodiesel, fatores como impurezas residuais e o teor de ácidos graxos são responsáveis pela maior corrosividade desse biocombustível (CURSARU et al., 2014; GU et al., 2018; FAZAL et al., 2016).

Diferentes pesquisas vêm sendo realizadas no sentido de avaliar a resistência a corrosão de diferentes tipos de ligas metálicas em contato com biodiesel provenientes de fontes vegetais diversas (GU et al., 2018; CHANDRAN et al., 2016; KOVÁCS et al., 2015; MARU et al., 2009). Fazal et al (2016) observaram que o ferro fundido apresenta uma maior taxa de corrosão em comparação com um aço de baixo carbono após imersão em biodiesel de palma. Jin et al. (2015) investigaram a resistência a corrosão de um aço carbono (ASTM 1045) em contato com biodiesel de palma, e observaram que a taxa de corrosão aumentou com a temperatura do meio e com o tempo de imersão. Cursaru et al (2014) verificaram que as ligas de alumínio apresentam maior resistência a corrosão em comparação com o cobre e o aço carbono após imersão em biodiesel de girassol. De modo semelhante, Chew et al. (2013) observaram que as ligas de magnésio apresentam uma resistência à corrosão consideravelmente menor que as de alumínio após exposição em biodiesel de palma. No entanto, apesar do crescimento de pesquisas a respeito do tema, ainda existem aspectos que tratam da composição química do biodiesel e sua relação com a corrosividade desse material que necessitam de maiores esclarecimentos.

Considerando a crescente aplicação das ligas de alumínio e o avanço da indústria dos biocombustíveis, esse trabalho tem como objetivo avaliar a composição química do biodiesel de soja em termos de teor de ácidos graxos e a interação desse biocombustível com as ligas de alumínio 6061 e 5052. 


\section{MATERIAL E MÉTODOS}

As ligas metálicas utilizadas neste trabalho consistiram de ligas comerciais da classe 6061 e 5052 (H38) cuja composição química está listada na Tabela 1.

Tabela 1 - Composição química das ligas metálicas.

\begin{tabular}{ccc}
\hline Composição Química (\%) & Liga 5052 & Liga 6061 \\
\hline Silício & 0,173 & 0,631 \\
Ferro & 0,224 & 0,333 \\
Cobre & 0,00287 & 0,252 \\
Manganês & 0,0417 & 0,0526 \\
Magnésio & 2,34 & 0,800 \\
Zinco & 0,0176 & 0,011 \\
Níquel & 0,00579 & $<0,005$ \\
Cromo & 0,164 & 0,0457 \\
Chumbo & $<0,005$ & 0,00876 \\
Estanho & 0,0216 & 0,00544 \\
Titânio & 0,0218 & 0,0101 \\
Alumínio & Balanço & Balanço \\
\hline
\end{tabular}

Fonte: Os autores.

A avaliação da corrosividade do biodiesel de soja foi realizada mediante ensaios de imersão de acordo com as Normas ASTM G1 e ASTM G31. Para a imersão no biodiesel de soja as amostras foram cortadas em formato retangular nas dimensões de $1,5 \times 3,0 \mathrm{~cm}$, conforme esquema apresentado na Figura 1.

Figura 1 - Amostras das ligas de alumínio para ensaios de imersão.

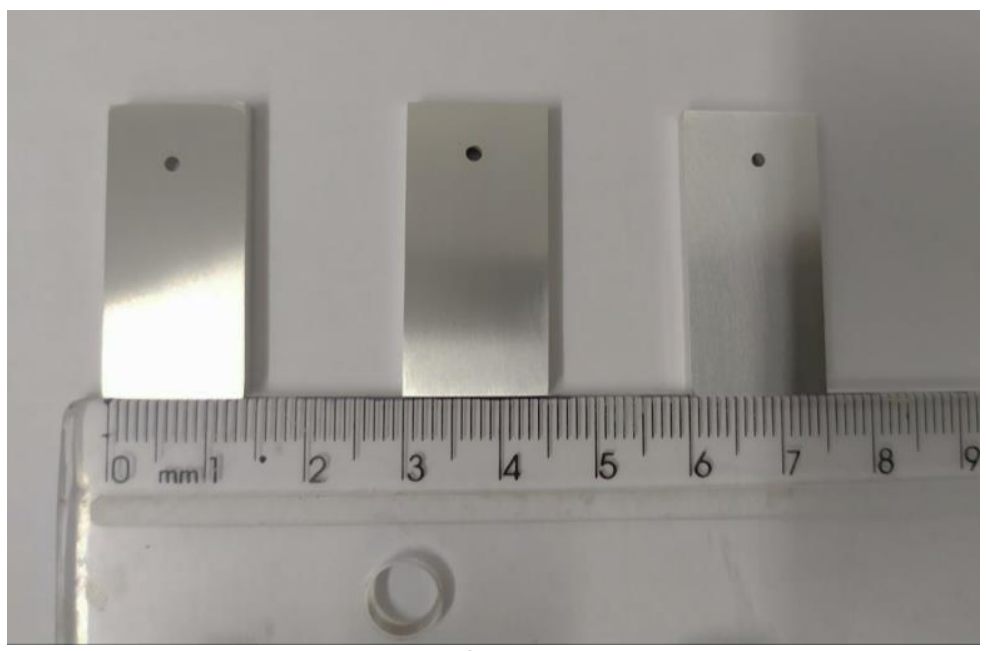

Fonte: Os autores.

Após o corte, as amostras foram lixadas com auxílio de uma politriz de bancada (AROTEC - modelo AROPOL VV) e lixas d'água com diferentes granulometrias (100, 200, 300, 400 e 600). Em seguida, as amostras foram lavadas em água destilada, desengorduradas com álcool e solução de hidróxido de sódio, enxaguadas em água destilada e secas com jato de ar frio.

Após limpeza, as amostras metálicas foram pesadas em balança analítica com 
precisão de $0,0001 \mathrm{~g}$. A pesagem das amostras antes e após os ensaios de imersão foi realizada até obtenção de uma triplicata do valor obtido. Desta maneira, foram definidas as massas iniciais de cada amostra para posterior cálculo dos valores de perda de massa e da taxa de corrosão. Os ensaios de imersão foram conduzidos em temperatura ambiente e sem agitação (imersão estática), conforme indicado na Figura 2.

A imersão das ligas de alumínio no biodiesel de soja foi realizada, com e sem vedação do béquer, a fim de verificar a relação entre o teor de água absorvido pelo biodiesel e a corrosividade do mesmo.

O teor de ácido graxo do biodiesel também foi avaliado, foram preparadas três amostras B0 (biodiesel puro), B10 (10\% de ácido oleico) e B20 (20\% de ácido oleico). A acidez das amostras foi determinada antes da imersão de acordo com a Norma EM 14104

O método EN 14104 (COMITE EUROPEEN DE NORMALISATION, 2003) utiliza uma solução alcoólica de hidróxido de potássio $(\mathrm{KOH})$ como titulante e fenolftaleína como indicador, sendo o resultado expresso em $\mathrm{mg} \mathrm{KOH} / \mathrm{g}$ de óleo. A norma estabelece um limite máximo de acidez de $0,5 \mathrm{mg}$ de $\mathrm{KOH}$ por $\mathrm{g}$ de óleo ou $3 \%$ de ácido oleico.

Figura 2 - Imersão das amostras em triplicata em biodiesel de soja.

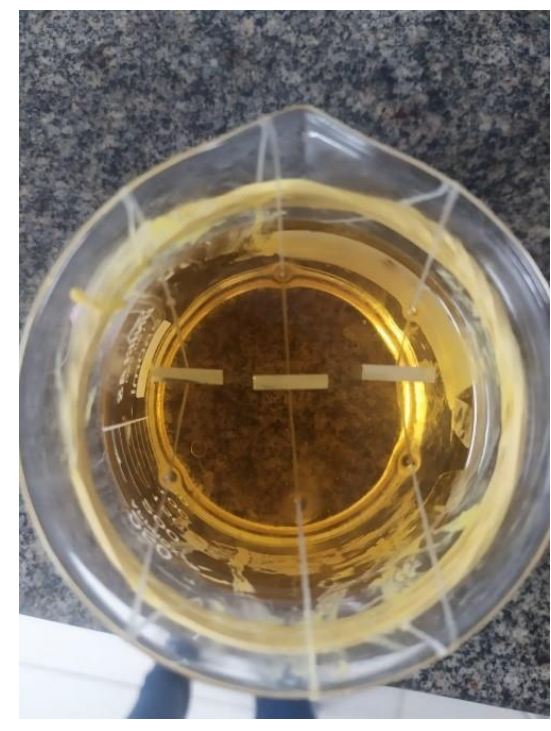

Fonte: Os autores.

O comportamento das amostras metálicas durante a imersão foi monitorado em intervalos específicos de tempo: 168, 720, 1440 e 2160 horas para as amostras de biodiesel puro (B0). As amostras com diferentes teores de ácido oleico - B10 e B20 foram monitoradas por 168 e 720 horas. A cada retirada, as amostras foram lavadas, desengorduradas e pesadas. A pesagem de cada uma das amostras foi realizada até a obtenção de uma triplicata. A perda de massa (em gramas) para cada amostra foi obtida através das diferenças entre as massas antes e após a imersão. A quantidade de biodiesel utilizada em cada ensaio foi quantificada respeitando a solicitação da norma, 
que indica uma quantidade de fluido de $0,20 \mathrm{~mL} / \mathrm{mm}^{2}$ de amostra.

A partir dos resultados da perda de massa, foram calculadas as taxas de corrosão das amostras de acordo com a Equação 1.

$$
\text { Taxa de Corrosão }=\frac{k \cdot W}{A \cdot t \cdot \rho}
$$

Onde:

$\mathrm{K}=$ constante tabelada pela ASTM G31 (87600 mm/ano);

$W=$ perda de massa, em gramas, para ensaios de corrosão generalizada;

$\mathrm{A}=$ área da amostra em $\mathrm{cm}^{2}$;

$\mathrm{t}$ = tempo de exposição, em horas e

$\rho=$ densidade da amostra, em $/ \mathrm{cm}^{3}$.

\section{RESULTADOS E DISCUSSÃO}

Os resultados obtidos a partir dos ensaios de imersão podem ser verificados nas Figuras 3 a 4, que apresentam as taxas de corrosão em função do tempo para as ligas 5052 e 6061 imersas em biodiesel de soja puro (B0) com e sem exposição ao ar.

Figura 3 - Taxa de corrosão da liga 5052 imersa em biodiesel de soja puro com e sem exposição ao ar.

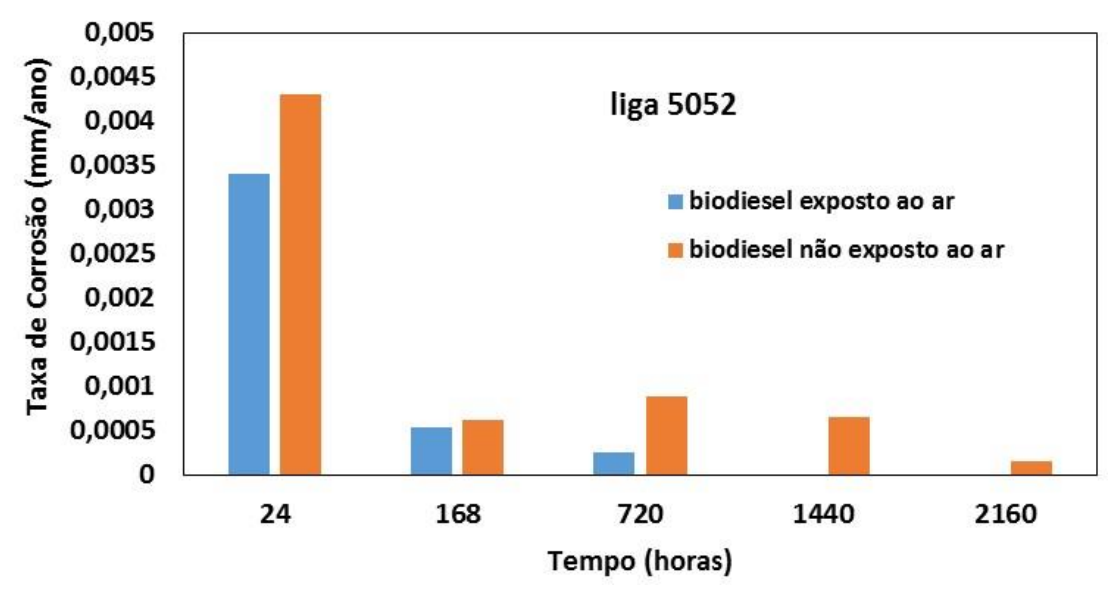

Fonte: Os autores.

É possível verificar que a taxa de corrosão é mais acentuada nas primeiras 24 horas de imersão. Após esse período, a taxa começa a diminuir e permanece praticamente constante nos primeiros trinta dias de imersão (720 horas). Nota-se que as amostras imersas no biodiesel, não exposto ao ar (recipiente fechado), apresentam uma maior taxa de corrosão levemente mais acentuada do que as amostras imersas no biodiesel exposto ao ar. Essa comparação foi realizada nas primeiras 720 horas de 
imersão, uma vez que não se observou perda de massa para a liga 5052 na condição biodiesel exposto ao ar para tempos mais longos (1440 e 2160 horas).

Figura 4 - Taxa de corrosão da liga 6061 imersa em biodiesel de soja puro com e sem exposição ao ar.

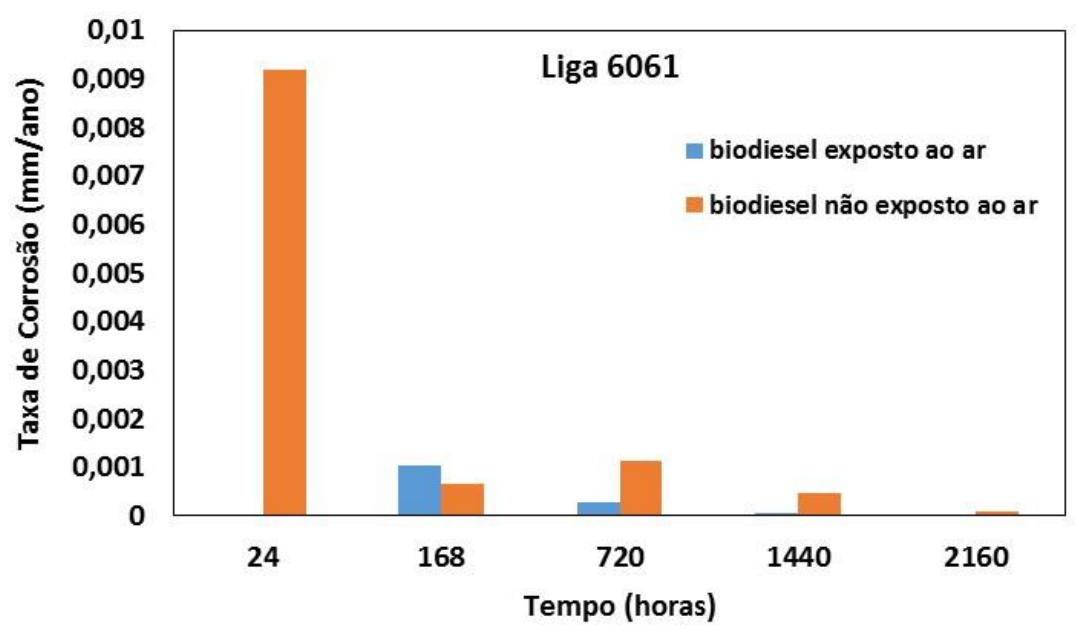

Fonte: Os autores.

A Figura 4 apresenta a taxas de corrosão para a liga 6061 imersa em biodiesel de soja puro, com e sem exposição ao ar atmosférico. De modo semelhante à liga 5052, a taxa de corrosão observada para a liga 6061 foi mais significativa nas primeiras 24 horas de imersão e para as amostras imersas em biodiesel não exposto. A Figura 5 apresenta uma comparação entre as taxas de corrosão das ligas 5052 e 6061 ambos na condição biodiesel exposto ao ar. Essa condição foi escolhida pois apresentou a maior taxa de corrosão para as duas ligas.

Figura 5 - Comparação das taxas de corrosão das ligas 5051 e 6061 imersas em biodiesel de soja puro exposto ao ar.

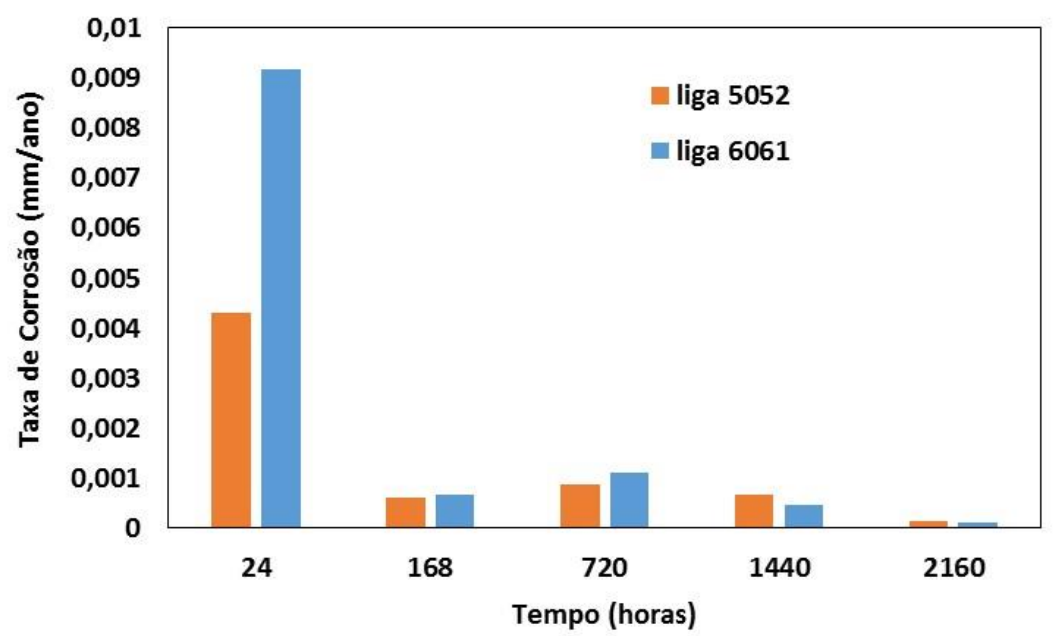

Fonte: Os autores.

Verifica-se, claramente, que para ambas as ligas, a taxa de corrosão é mais 
acentuada nas primeiras 24 horas de imersão. Nesta situação se observa que a liga 6061 apresenta uma taxa de corrosão duas vezes mais elevada que a liga 5052. Para tempos mais logos, a taxa diminui e torna-se praticamente a mesma para ambas as ligas. Esse comportamento inicial pode estar relacionado com a composição química das ligas. A liga 5052 apresenta maior teor de cromo e titânio, o que poderia estar relacionado com a menor taxa de corrosão apresentada por esse material nas primeiras horas de imersão. Após esse período ocorreria a formação do óxido, promovendo uma maior proteção a superfície e provocando uma queda da taxa de corrosão. Após a formação do óxido, a taxa de corrosão se torna muito próxima para ambos os materiais. Um comportamento similar foi observado por Hu et al. (2012), ao verificarem quem em comparação com cobre e aço carbono, o alumínio e o aço inoxidável apresentaram as menores taxas de corrosão. As ligas de alumínio e aço são propícias à formação de óxidos que impossibilitam a continuação da oxidação do metal pois dificultam a interação do oxigênio com a superfície metálica e também a interação do metal com o biocombustível.

O efeito do teor de ácido oleico (ácido graxo) sobre a corrosividade do biodiesel pode ser observado na Figura 6, que apresenta as taxas de corrosão para a liga 6061 imersa em biodiesel puro (B0), biodiesel $+10 \%$ de ácido oleico (B10) e biodiesel $+20 \%$ de ácido oleico (B20). As perdas de massa nessas condições foram aferidas após 168 e 720 horas de imersão.

Figura 6 - Comparação das taxas de corrosão da liga 6061 imersa em biodiesel de soja puro (B0), biodiesel $+10 \%$ ácido oleico (B10) e biodiesel $+20 \%$ ácido oleico (B20).

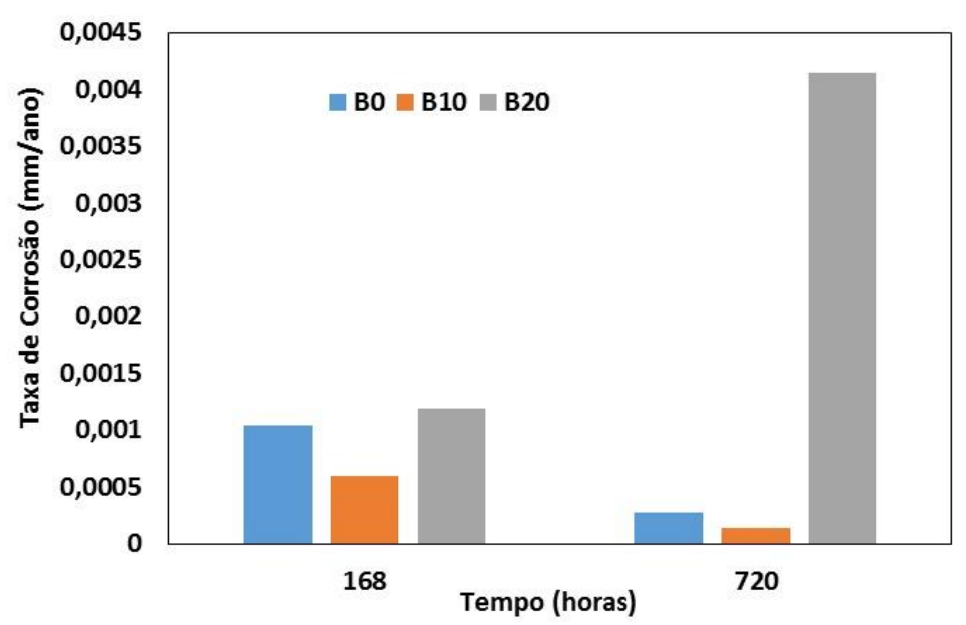

Fonte: Os autores.

Os resultados obtidos mostram que o teor de ácido oleico no biodiesel apresenta uma influência na resistência à corrosão da liga de alumínio 6061. Observa-se que um teor de $20 \%$ de ácido oleico promoveu um aumento da corrosividade do biodiesel suficiente para aumentar a taxa de corrosão. O aumento da taxa de corrosão no caso do B20 torna-se ainda mais significativa para maiores tempos de imersão. 


\section{CONCLUSÃO}

Considerando os resultados dos experimentos, que foram realizados com o objetivo de avaliar a resistência à corrosão de duas ligas comerciais de alumínio em contato com biodiesel de soja, é possível concluir que:

- Ambas as ligas 6061 e 5052 apresentam maiores taxas de corrosão nas horas iniciais de imersão, a taxa mostra uma considerável diminuição com o aumento do tempo de imersão. Esse comportamento pode estar associado com a formação do óxido de alumínio sobre a superfície do material que apresenta um efeito protetor e promove a redução das taxas de corrosão.

- Para as primeiras horas de imersão, a liga 5052 apresenta uma maior resistência à corrosão em comparação à liga 6062, ambas imersas em biodiesel puro. Esse comportamento pode estar relacionado à composição química, uma vez que as liga 5052 apresenta maior teor de cromo e de titânio. Para maiores tempos de imersão não se observam diferenças significativas entre as taxas de corrosão das duas ligas.

- O efeito do teor de ácido oleico sobre a corrosividade do biodiesel de soja e a resistência à corrosão da liga de alumínio 6061 mostrou que o efeito do ácido é mais significativo para a maior concentração avaliada ( $20 \%$ de ácido oleico). Foi observado que essa concentração promoveu um aumento da taxa de corrosão da liga, e esse aumento torna-se mais significativo para maiores tempos de imersão.

\section{AGRADECIMENTOS}

Os autores agradecem à Fundação de Amparo à Pesquisa do Estado do Rio de Janeiro - FAPERJ pelo financiamento das pesquisas, dos laboratórios e pelas bolsas de Iniciação Científica e Tecnológica concedidas. À Fundação Centro Universitário Estadual da Zona Oeste - UEZO. À SH Indústria de Metalurgia e Serviços Ltda. por fornecer as amostras metálicas para realização dos ensaios.

\section{REFERÊNCIAS}

ALUMICUPPER. Catálogo de Produtos de Alumínio. Disponível em: https://www.alumicopper.com.br/pdf/catalogo produtos alumicopper.pdf. Acesso em: 15 out. 2019.

ALVES, S.M.; DUTRA-PEREIRA, F.K.; BICUDO, T.C. Influence of stainless steel corrosion on biodiesel oxidative stability during storage. Fuel. v. 249, p. 73-79, 2019.

ASSOCIAÇÃO BRASILEIRA DO ALUMÍNIO - ABAL. Alumínio brasileiro: soluções para uma vida sustentável /Associação Brasileira do Alumínio - São Paulo: Associação Brasileira do Alumínio, 2017. Disponível em: http://abal.org.br/downloads/publicacoes/aluminio-brasileiro-solucoes-para-uma-vidasustentavel-port.pdf. Acesso em: 15 out. 2019. 
ASTM G1-90, Standard Practice for Preparing, Cleaning, and Evaluation Corrosion Test Specimens. ASTM International, 1990.

ASTM G31-72, Standard Practice for Laboratory Immersion Corrosion Testing of Metals. ASTM International, 2004.

CHANDRAN, D. et al. Investigation of the effects of palm biodiesel dissolved oxygen andconductivity on metal corrosion and elastomer degradation under novel immersion method. Applied Thermal Engineering, v. 104. p. 294-308, 2016.

CHEW, K.V. et al. Corrosion of magnesium and aluminum in palm biodiesel: A comparative evaluation. Energy, v. 57, p. 478-483, 2013.

COMITE EUROPEEN DE NORMALISATION. EN 14104: Fat and Oil Derivatives Fatty Acid Methyl Esters (FAME), Determination of Acid Value, Berlin: European Committee for Standardization, 2003.

CURSARU, D. L. et al. Degradation of automotive materials upon exposure to sunflower biodiesel. Industrial Crops and Products, v. 54. p. 149-158, 2014.

DEYAB, M. A. et al. Improving the sustainability of biodiesel by controlling the corrosive effects of soybean biodiesel on aluminum alloy 5052 H32 via cardanol. Industrial Crops \& Products, v. 130, p. 146-150, 2019.

FAZAL, M. A.; HASEEB, A. S. M. A.; MASJUKI, H. H., Effect of different corrosion inhibitors on the corrosion of cast iron in palm biodiesel. Fuel Processing Technology, v. 92, n. 11, p. 2154-2159, 2011.

FAZAL, M. A. et al. Inhibition study of additives towards the corrosion of ferrous metal in palm biodiesel. Energy Conversion and Management, v. 122, p. 290-297, 2016.

FERNANDES, D. M. et al. Storage stability and corrosive character of stabilised biodiesel exposed to carbon and galvanised steels. Fuel, v. 107. p. 609-614, 2013.

GENTIL, V. Corrosão. 5. ed. Rio de Janeiro: LTC, 2007.

GU, J. et al. Biodiesel production from palm oil and mixed dimethyl/diethyl carbonate with controllable cold flow properties. Fuel, v. 216, p. 781-786, 2018.

$\mathrm{HU}$, E. et al. Corrosion behaviors of metals in biodiesel from rapeseed oil and methanol. Renewable Energy, v. 37. n. 1. p. 371-378, 2012.

JIN, D. et al. Corrosion behavior of ASTM 1045 mild steel in palm biodiesel. Renewable Energy, v. 81, p. 457-463, 2015.

$\mathrm{KOCH}$, G. et al. Corrosion Costs and Preventive Strategies in the United States. Nace International,n. FHWA-RD-01-156, 2006. Disponível em:

https://www.nace.org/uploadedFiles/Publications/ccsupp.pdf. Acesso em: 15 out. 2019.

$\mathrm{KOCH}$, G. et al. International Measures of Prevention, Application, and Economics of Corrosion Technologies Study. Nace International Impact, 2016. Disponível em: http://impact.nace.org/documents/Nace-International-Report.pdf. Acesso em: 15 out. 2019. 
KOVÁCS, A. et al. Aspects of storage and corrosion characteristics of biodiesel. Fuel Processing Tecnology, v.134, p. 59-64, 2015.

MARU, M. M. et al. Biodiesel compatibility with carbon steel and HDPE parts. Fuel Processing Tecnology, v.90. n. 9. p. 1175-1182, 2009.

NUNES, L. P. Fundamentos de Resistência à Corrosão. Rio de Janeiro: Editora Interciência, 2007.

OZTURK, F.; TOROS, S.; KILIC, S., Evaluation of tensile properties of 5052 type aluminum-magnesium alloy at warm temperatures. Archives of Materials Science and Engineering, v. 34, n. 2, p. 95-98, 2008. 